\title{
Estudio sobre el trabajo del estudiante en la adaptación al Espacio Europeo de Educación Superior a través del indicador DAA (dedicación alumno-asignatura)
}

\author{
Eva Lantarón, Rita Romero-Galisteo, Pablo Gálvez, Teresa Labajos
}

Introducción. Uno de los objetivos del proceso de Bolonia es mejorar la calidad de la enseñanza y ello ha supuesto un cambio en la orientación de la actividad docente, con una mayor carga de trabajo para el profesorado y para el estudiante al requerir una mayor autonomía mediante trabajo no presencial. De esta manera, se antoja necesario contemplar la información que facilita el alumnado, de forma directa o mediante la recogida y posterior transformación de datos en indicadores, proporcionando al profesorado universitario una herramienta para la mejora de la organización de actividades en las materias impartidas.

Sujetos y métodos. Diseño observacional de corte transversal y tipo descriptivo, con una muestra compuesta por 116 estudiantes de fisioterapia de la Universidad de Vigo (curso académico 2007-2008). El objetivo de este trabajo es la construcción de un indicador del esfuerzo requerido para cada asignatura, cuya aplicación resulte pragmática y fiable para señalar asignaturas donde la relación entre carga de trabajo y créditos asignados es significativa con respecto a las demás asignaturas.

Resultados. Se comprobó que sólo el 18,51\% de las asignaturas impartidas en el Grado de Fisioterapia están planificadas acorde al número de créditos ECTS asignados en la planificación docente.

Conclusiones. El indicador que se presenta, al basarse en los residuos de un modelo de regresión individual para cada estudiante, se muestra independientemente porque no toma en consideración respuestas poco fidedignas, por lo que ayudará al profesorado a una mejor planificación docente.

Palabras clave. Carga de trabajo. Espacio Europeo de Educación Superior. Planificación docente. Profesorado universitario.

Study of the student's work in the adaptation to the European Area of Higher Education through the indicator 'dedication-student-subject' (DAA)

Introduction. One of the objectives of the Bologna process is to improve the quality of teaching and this has meant a change in the orientation of teaching, with a greater workload for both teacher and student by requiring greater autonomy through non-presential work. It seems necessary to contemplate information that facilitates students, directly or through the collection and subsequent transformation of data on indicators, providing a tool for the improvement of the organization's activities in the subjects taught to the faculty.

Subjects and methods. Through a cross-sectional and descriptive observational design, a sample of 116 Physiotherapy students at the University of Vigo (academic year 2007-2008). The aim of this paper was the construction of an indicator of the effort required for each subject, which is pragmatic and reliable to designate subjects where the relationship between workload and assigned credits are meant with respect to the other subject.

Results. Only the $18.51 \%$ of the subjects in the degree of Physical Therapy are planned according to the number of ECTS credits allocated in the educational planning.

Conclusions. The indicator that is presented, based on the waste of a regression model individual for each student, shown independently since not taking into account some credible answers, so it will help teachers to better teaching planning.

Key words. European Higher Education Area. Professor. Teacher planning. Workload.
Departamento de Biología Funcional y Ciencias de la Salud; Facultad de Fisioterapia; Universidade de Vigo; Pontevedra (E. Lantarón). Departamento de Psiquiatría y Fisioterapia; Facultad de Ciencias de la Salud; Universidad de Málaga (R. Romero-Galisteo, T. Labajos). Universidad Internacional de La Rioja; Logroño, España (P. Gálvez).

Correspondencia:

Dra. Eva M. Lantarón Caeiro. Departamento de Biología Funcional y Ciencias de la Salud. Facultad de Fisioterapia. Universidade de Vigo. Campus A Xunqueira, s/n. E-36005 Pontevedra.

Fax: +34 986801780

E-mail:

evalantaron@uvigo.es

Conflicto de intereses: No declarado.

Competing interests: None declared.

(C) 2015 FEM 


\section{Introducción}

Desde que en 1999 se firmara la Declaración de Bolonia, las universidades europeas están inmersas en el denominado Espacio Europeo de Educación Superior (EEES). Es en el año 2010 cuando se impide a las universidades de nuestro país la matriculación de nuevos alumnos en las antiguas titulaciones, cuya estructura tradicional queda obsoleta con la dimensión europea [1]. La adaptación de titulaciones y modos de impartir la enseñanza, con nuevos retos y exigencias, producen situaciones en las que predomina la falta de coordinación y sistematización, prevaleciendo la diversidad y la divergencia [2].

Uno de los aspectos claves de la reforma es el establecimiento de un sistema de créditos ECTS ( $E u$ ropean Credit Transfer System) basados en el esfuerzo efectivo o carga de trabajo que un estudiante medio requiere para superar la asignatura, frente al modelo tradicional que tiene en cuenta exclusivamente el número de horas de clase [3]. Por otro lado, los docentes se han visto inmersos en un proceso de cambio que afecta a la manera de concebir la enseñanza respecto a la estructura de las materias que imparten [2]. En esta línea, el profesorado debe saber orientar su docencia hacia un cambio en la actitud tradicionalmente pasiva del alumnado a otra de aprendizaje autónomo y activo [4]. Nos hallamos, por tanto, ante un momento de reflexión sobre el proceso de enseñanza-aprendizaje y los resultados que se derivan de éste [5], donde la figura del docente sigue siendo el eje central y uno de los componentes decisivos de cualquier proceso de formación [6].

Diversas investigaciones surgidas en la última década se han centrado en la calidad dentro de la educación superior $[7,8]$. El interés que despierta su análisis hace que el estudio sobre el rendimiento académico de los estudiantes $[9,10]$, las políticas de evaluación del desempeño [11], las teorías del aprendizaje [12] o el engagement y logro académico [6,13] sean, entre otros aspectos, objeto de debate en la literatura científica.

No obstante, dentro de las cinco grandes funciones a desarrollar por los profesores universitarios [14, 15], el diseño y planificación de los programas de las asignaturas impartidas representa un aspecto de cierta complejidad al existir gran variabilidad de horas dedicadas al estudio entre los alumnos [16]. Por otro lado, la dificultad de una asignatura depende en gran medida de la planificación docente y del número de horas que el alumnado debería estudiar según la asignación de créditos ECTS. Esto pone de manifiesto la dificultad de establecer el cómputo de créditos para las distintas asignaturas, siendo necesario contemplar la información que facilita el alumnado mediante la recogida y posterior transformación de dicha información en indicadores que ayudarán a mejorar la organización de actividades en las materias impartidas, además de suponer una adecuada herramienta para conseguir una optimización en la relación coste-beneficio, pues la orientación fundamental pasa por tratar que los estudiantes logren su titulación en los tiempos estipulados [9].

Por ello resulta prioritario identificar, mediante el uso de indicadores, cuáles son las asignaturas que requieren un mayor esfuerzo por parte del alumnado con respecto a lo planificado, y en este sentido la construcción de indicadores permite una aproximación a una realidad académica difícilmente observable y cuantificable de otra manera.

El objetivo de este estudio es la construcción de un indicador del esfuerzo requerido para cada asignatura que pueda utilizarse para señalar asignaturas donde la relación entre carga de trabajo y créditos asignados es significativa con respecto a las demás asignaturas, en situaciones donde la carga de trabajo tiene una medición poco fiable.

\section{Sujetos y métodos}

Diseño no experimental, de corte transversal y de carácter descriptivo. Se empleó, por un lado, una variable de medida de carácter objetivo, en este caso la relación existente entre crédito-asignaturas, es decir, el número de créditos ECTS asignados en la programación docente a cada asignatura, y por otro, una variable subjetiva; en este caso, para cada combinación de estudiante y asignatura, se preguntó el número de horas dedicado a preparar cada una de ellas.

\section{Participantes}

Se seleccionaron, mediante muestreo intencionado, 116 alumnos de segundo curso, tercer curso y recién egresados de la Escuela Universitaria de Fisioterapia de Pontevedra (Universidad de Vigo), pertenecientes al curso académico 2007-2008, de los que $45(38,8 \%)$ pertenecían a segundo curso, $37(31,9 \%)$ a tercer curso y $34(29,3 \%)$ eran recién egresados.

\section{Instrumento}

Se empleó el cuestionario de valoración de las cargas de trabajo de los estudiantes, utilizado en el Programa de Convergencia Europea ANECA 2003, para elaborar el Libro Blanco del Título de Grado en 
Fisioterapia [17]. El cuestionario se desarrolló adaptando los modelos utilizados por el proyecto Tuning Educational Structures in Europe [18]. Esta herramienta se compone de cinco ítems, encaminados por la frase 'estimación en horas de la dedicación del alumno': requeridas de examen, requeridas de tutoría, requeridas de estudio, otras actividades y totales. El uso de este cuestionario ayuda a los participantes a descubrir el patrón de dedicación de los alumnos a las asignaturas, cumplimentando un cuestionario por cada asignatura superada en el curso anterior.

\section{Procedimiento}

Para la recogida de datos, se seleccionó el curso académico 2007-2008 porque la titulación puso en marcha en el curso 2005-2006 las líneas de actuación dirigidas a experiencias de implantación de nuevas metodologías de aprendizaje y evaluación en el primer curso completo de la titulación de fisioterapia, por lo que en el 2007-2008 estaban introducidos los tres cursos en el proyecto piloto del EEES y, de esta manera, las asignaturas estaban adaptadas al mismo. Así, las asignaturas de primer curso fueron valoradas por alumnos de segundo; las asignaturas de segundo curso, por alumnos de tercero, y los recién egresados evaluaron las asignaturas de tercer curso. La distribución de asignaturas evaluadas por curso se exponen en la tabla.

Los participantes contestaron el cuestionario de forma individual, siendo previamente informados tanto del objetivo de la investigación como de la participación voluntaria en ésta. Se enfatizó sobre la confidencialidad de los datos y la honestidad en las respuestas, y tras la recogida del cuestionario se verificó que estuviera correctamente cumplimentado para evitar valores perdidos. A los alumnos de segundo y tercer curso se les pasó el cuestionario en clase, y a los alumnos egresados, vía correo electrónico o correo postal. Previamente se contactó vía telefónica con cada uno de los egresados.

\section{Análisis de datos}

Para cada estudiante se ajusta un modelo de regresión que pone la medición del esfuerzo declarado por éste como un modelo de regresión creado para el individuo a partir de los créditos ECTS de las asignaturas:

$$
\begin{gathered}
\text { Esfuerzo }_{i, A_{i_{k}}}=b_{0 i}+b_{1 i} \text { ECTS }_{A_{i_{k}}}+\text { residuo }_{i, A_{i_{k}}} \\
\forall A_{i_{k}} \in\left\{A_{i_{1}}, A_{i_{2}} \ldots A_{i_{n_{i}}}\right\}
\end{gathered}
$$

\begin{tabular}{|c|c|c|c|}
\hline & Tipo & Asignatura & Créditos ECTS \\
\hline \multirow{9}{*}{ Primer curso } & Troncal & Anatomía Humana & 10,5 \\
\hline & Troncal & Ciencias Psicosociales Aplicadas & 6,0 \\
\hline & Troncal & Fisiología Humana & 7,5 \\
\hline & Troncal & Fisioterapia General I & 9,0 \\
\hline & Troncal & Fisioterapia General II & 9,0 \\
\hline & Troncal & Fisioterapia General III & 9,0 \\
\hline & Troncal & Salud Pública y Legislación Sanitaria & 7,5 \\
\hline & Obligatoria & Biofísica y Bioquímica & 4,5 \\
\hline & Obligatoria & Radiología & 4,5 \\
\hline \multirow{7}{*}{ Segundo curso } & Troncal & Afecciones Médicas I & 6,0 \\
\hline & Troncal & Afecciones Quirúrgicas I & 6,0 \\
\hline & Troncal & Estancias Clínicas I & 12,0 \\
\hline & Troncal & Fisioterapia Manipulativa Articular & 9,0 \\
\hline & Troncal & Fisioterapia Neurológica y Especial & 12,0 \\
\hline & Troncal & Fisioterapia Respiratoria y Masoterapia & 6,0 \\
\hline & Obligatoria & Movimiento Funcional en Fisioterapia & 6,0 \\
\hline \multirow{5}{*}{ Tercer curso } & Troncal & Afecciones Médicas II & 6,0 \\
\hline & Troncal & Afecciones Quirúrgicas II & 6,0 \\
\hline & Troncal & Estancias Clínicas II & 12,0 \\
\hline & Troncal & Fisioterapia en Especialidades Clínicas I & 12,0 \\
\hline & Troncal & Fisioterapia en Especialidades Clínicas II & 12,0 \\
\hline \multirow{6}{*}{$\begin{array}{l}\text { Segundo y } \\
\text { tercer curso }\end{array}$} & Opcional & Fisioterapia Deportiva & 4,5 \\
\hline & Opcional & Fisioterapia en Atención Primaria & 4,5 \\
\hline & Opcional & Fisioterapia en Geriatría & 4,5 \\
\hline & Opcional & Introducción a la Farmacología & 4,5 \\
\hline & Opcional & Introducción a la Fisiopatología & 4,5 \\
\hline & Opcional & Teoría de Cuidados & 4,5 \\
\hline
\end{tabular}

Tabla. Relación de asignaturas evaluadas por curso, tipo y número de créditos. 
Figura. Valores del indicador, media y desviación estándar.

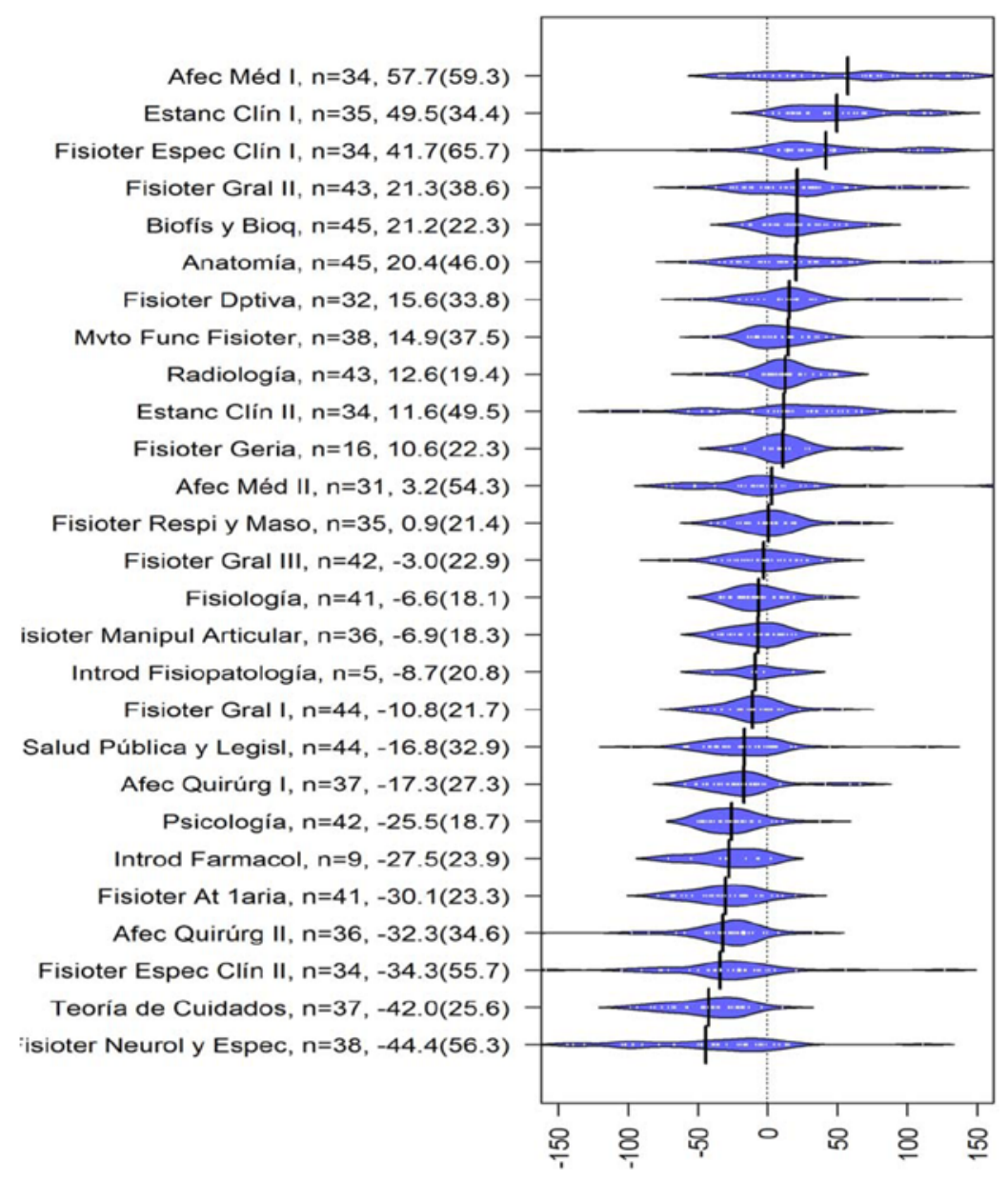

donde:

$$
\sum_{k=1}^{n_{i}} \text { residuo }_{i, A_{i_{k}}}=0
$$

Así, el indicador que se muestra se basa en los residuos de un modelo de regresión individual para cada estudiante, que dan por término medio un valor cero. El valor que muestra el indicador es independiente de la capacidad de cada alumno, de su memoria o del mayor o menor grado de fidelidad con el que haya respondido.

La puntuación que una asignatura recibe de un estudiante $E_{i}$ es una cantidad que representa cuánto se desvía el número de horas que el estudiante recuerda haber dedicado a ella con respecto a la cantidad estimada por un modelo de regresión ajustado individualmente para dicho estudiante, usando como variable independiente los créditos ECTS de cada asignatura en la que se hubiese matriculado.

De esta manera, si una asignatura recibe una puntuación cero por parte de un estudiante significa que recuerda haber dedicado un esfuerzo a ella que está en consonancia con la relación entre créditos y esfuerzo recordado visto en las demás asignaturas. Una puntuación positiva refleja que, para esa asignatura en particular, el estudiante recuerda haberle dedicado mayor esfuerzo del que le correspondería de acuerdo con el patrón del resto de asignaturas.

El análisis de datos se llevó a cabo mediante el uso del programa $\mathrm{R}$ [19] y el código para su uso se puede descargar a través de la página web http://webs. uvigo.es/lanfis/daa/.

\section{Resultados}

En base a los análisis realizados, cuyos resultados se muestran en la figura, el indicador propuesto muestra un valor próximo a cero tan sólo en la asignatura Fisioterapia Respiratoria y Masoterapia (0,9 \pm $21,4)$, es decir, el número de créditos ECTS asignados se ajusta a la carga de trabajo que cada estudiante ha manifestado haber dedicado para la superación de la misma, considerando por tanto que ha existido una planificación docente adecuada. Afecciones Médicas II $(3,2 \pm 54,3)$, Fisioterapia General III $(-3 \pm 22,9)$, Fisiología $(-6,6 \pm 18,1)$ y Fisioterapia Manipulativa Articular $(-6,9 \pm 18,3)$ mostraron valores cercanos a cero.

Existen asignaturas donde se pone de manifiesto que los estudiantes han dedicado más tiempo del inicialmente previsto, según el número de créditos asignados, como sería el caso de Fisioterapia Geriátrica $(10,6 \pm 22,3)$, Estancias Clínicas II $(11,6 \pm 49,5)$, Radiología (12,6 $\pm 19,4)$, Movimiento Funcional en Fisioterapia $(14,9 \pm 19,4)$, Fisioterapia Deportiva $(15,6 \pm 33,8)$, Anatomía $(20,4 \pm 46)$, Biofísica y Bioquímica (21,2 $\pm 22,3)$, Fisioterapia General II (21,3 \pm 38,6), Fisioterapia en Especialidades Clínicas I $(41,7 \pm 65,7)$, Estancias Clínicas I $(49,5 \pm 34,11)$ y Afecciones Médicas I (57,7 $\pm 59,3)$.

Los estudiantes manifestaron en cambio haber dedicado menos tiempo del previsto, según los valores obtenidos del indicador que se muestra en la figura, para las asignaturas Introducción a la Fisiopatología $(-8,7 \pm 20,8)$, Fisioterapia General I $(-10,8$ $\pm 21,7)$, Salud Pública y Legislación $(-16,8 \pm 32,9)$, Afecciones Quirúrgicas I $(-17,3 \pm 27,3)$, Psicología $(-25,5 \pm 18,7)$, Introducción a la Farmacología (-27,5 $\pm 23,9)$, Fisioterapia en Atención Primaria $(-30,1 \pm$ 23,3), Afecciones Quirúrgicas II $(32,3 \pm 34,6)$, Fisio- 
terapia en Especialidades Clínicas $(-34,3 \pm 55,7)$, Teoría de Cuidados $(-42 \pm 25,6)$ y Fisioterapia Neurológica y Especial $(-44,4 \pm 56,3)$.

De esta manera, se observa que sólo el 18,51\% de las asignaturas del Grado en Fisioterapia están planificadas acorde al número de créditos asignados a éstas. El restante $81,48 \%$ de las asignaturas están planificadas por debajo $(40,74 \%)$ o por encima $(40,74 \%)$ de los créditos asignados.

\section{Discusión}

La presente investigación surgió a raíz de la reforma adoptada por la universidad española con la implantación del sistema de créditos ECTS exigido en el marco del EEES. Su realización en el momento de la implantación del grado ofrece la posibilidad al profesor de detectar desde el inicio en qué medida su planificación docente se ajustaba al nuevo sistema de créditos, independientemente de la metodología utilizada.

En este estudio, se propone la utilización de un indicador para optimizar la asignación de créditos ECTS a cada una de las asignaturas contempladas en los distintos estudios de grado universitarios. Dicho indicador muestra si las horas que recuerda haber dedicado cada estudiante en particular están en consonancia con las que se esperan de acuerdo a lo que el alumno ha dedicado a otras asignaturas en relación a su número de créditos. De esta manera, y dado que los diversos procedimientos para determinar la carga de trabajo del estudiante que se proponen en la literatura científica resultan complejos y laboriosos $[18,20,21]$, la utilización del indicador permite realizar inferencias a través del estudio detallado de los diferentes casos.

Mediante el tratamiento estadístico empleado, se elimina tanto el sesgo de memoria como la influencia de las distintas variables que usualmente se han analizado, como el talento del estudiante, los conocimientos previos, la dificultad o grado de ansiedad que provoca una asignatura, la relación profesor-alumno, el entorno familiar, la metodología docente o incluso el nivel de exigencia del profesor.

Por estos motivos, este trabajo aporta una innovadora y pragmática herramienta que ayudará a la planificación docente del profesorado, en tanto se utilice el indicador aquí expuesto para optimizar la asignación de créditos ECTS a cada una de las asignaturas contempladas en los distintos estudios de grado.

La articulación organizativa en la universidad es percibida por parte del profesorado como un factor que debe reforzarse para dejar paso a un cambio relevante en la calidad de la formación universitaria [22], por lo que la propuesta que aquí se presenta podría servir actualmente para cubrir una de las demandas del profesorado, que reclama impulsar la coordinación de asignaturas y equipos docentes para disminuir la inversión en tiempo, carga docente, dedicación y complejidad de la docencia.

Finalmente, es necesario tener en cuenta algunas limitaciones del estudio. En primero lugar, sería conveniente ampliar el tamaño muestral, realizando un estudio multicéntrico y extendiéndolo a otros grupos de estudiantes de distintos grados universitarios. Los resultados obtenidos no son, por tanto, generalizables porque se llevó a cabo con una muestra de estudiantes de fisioterapia. De esta manera se facilitaría además la difusión de las experiencias docentes en cuanto al uso del indicador.

En segundo lugar, la naturaleza transversal del estudio impide establecer relaciones causales, por lo que se considera pertinente realizar estudios longitudinales y complementar las medidas autoinformadas del cuestionario con otros métodos de evaluación como, por ejemplo, entrevistas (tanto con el profesor como con los alumnos), utilizando así una metodología de investigación mixta. En este sentido, sería interesante replicar esta investigación enlazándola con los estudios que se están efectuando en torno a variables relacionadas con el engagement [13], surgiendo así una futura línea de investigación. De esta manera, se estaría dando un tiempo de adaptación tanto al profesor como al alumno.

Bibliografía

1. Rodríguez SM, Rodríguez F. Del crédito tradicional al crédito europeo (ECTS). Una experiencia piloto con alumnos del área de economía financiera y contabilidad. A Coruña: Tórculo; 2008

2. Hallbach A, Lázaro-Lafuente A, Pérez-Guerra J. La lengua inglesa en la nueva universidad española del EEES. Rev Educ 2013; 362: 105-32.

3. Jano MD, Ortiz S. Estimación del esfuerzo efectivo para superar la asignatura de estadística descriptiva en las licenciaturas de Economía y Administración de Empresa. Relieve: Revista Electrónica de Investigación y Evaluación Educativa 2007; 12: 191-202.

4. Ortiz VM, Jenaro C, García CC, Zubiauz MB, Mayor MA, Arana JM. Carga de trabajo en el EEES: la necesidad de coordinación docente entre asignaturas. Actas de las IX Jornadas de Redes de Investigación en Docencia Universitaria; 2011.

5. Santos MV, Vallelado E. Algunas dimensiones relacionadas con el rendimiento académico de estudiantes de administración y dirección de empresas. Univ Psicol 2013; 12: 737-52.

6. Küster I, Vila N. El docente universitario y sus efectos en el estudiante. Estad Educ 2012; 3: 157-82.

7. Hanne G, Askling B, Dittrich K, Froestad W, Haug P, Hofgaard K, et al. Assessing educational quality: knowledge production and the role of experts. Helsinki: ENQA; 2009.

8. Floriano-Pardal B, González-Jurado JA, Lucena-Cid IV, Molina-Díaz F, Naranjo-Gil D, Ruiz-Jiménez AM, et al. Seguimiento de los sistemas de garantía interna de calidad 
de los títulos de grado en la Universidad Pablo de Olavide In Bermúdez MP, Guillén-Riquelme A, comp. VIII Foro sobre Evaluación de la Calidad de la Investigación y de la Educación Superior: Libro de Capítulos. Granada: Asociación Española de Psicología Conductual; 2011.

9. Garbanzo-Vargas GM. Factores asociados al rendimiento académico en estudiantes universitarios. Una reflexión desde la calidad de la educación superior pública. Rev Educ 2007; 31: 43-63.

10. Pérez P, Nicolau JL. La teoría de las perspectivas en el rendimiento de los estudiantes. Rev Educ 2013; 360: 645-65.

11. Sisto V, Montecinos C, Ahumada-Figueroa L. Disputas de significado e identidad: la construcción local del trabajo docente en el contexto de las políticas de evaluación e incentivo al desempeño en Chile. Univ Psicol 2013; 12: 173-84.

12. Fryer LK, Ginns P, Walker RA, Nakao K. The adaptation and validation of the CEQ and the R-SPQ-2F to the Japanese tertiary environment. Br J Educ Psychol 2012; 84: 549-63.

13. Casuso-Holgado MJ, Cuesta-Vargas AI, Moreno-Morales N, Labajos-Manzanares MT, Barón-López FJ, Vega-Cuesta M. The association between academic engagement and achievement in health sciences students. BMC Med Educ 2013; 13: 33.

14. Butcher C, Davies C, Highton M. Designing learning from module outline to effective teaching. Abingdon: Routledge; 2006.
15. Zabalza MA. Competencias docentes del profesorado universitario: calidad y desarrollo profesional. Madrid: Narcea; 2003.

16. Tortella-Feliu M, Servera M, Llabrés J. Análisis de dos estrategias de enseñanza-aprendizaje en la docencia de terapia de conducta. Int J Clin Health Psychol 2006; 7: 547-58.

17. Rebollo J. Libro Blanco del Título de Grado en Fisioterapia. Madrid: ANECA; 2004.

18. González J, Wagenaar R. Tuning Educational Structures in Europe. Informe final. Fase uno. Bilbao: Universidad de Deusto; 2003.

19. R Development Core Team. R: a language and environment for statistical computing. Wien: R Foundation for Statistical Computing; 2007.

20. Chamber E. Work-load and the quality of student learning. Stud High Educ 1992; 17: 141-53.

21 Kember D. Interpreting student workload and the factors which shape student's perception of their workload. Stud High Educ 2004; 29: 165-84.

22. Tierno JM, Iranzo P, Barrios C. El compromiso organizativo e institucional para diseñar y evaluar competencias en la universidad. Rev Educ 2013; 361: 223-51. 